\title{
Neurodegeneration with brain iron accumulation
}

\author{
Neurodegeneração com acúmulo de ferro cerebral \\ João Carlos Papaterra Limongi
}

Universidade de São Paulo,

Faculdade de Medicina, Hospital das Clínicas, Departamento de

Neurologia, São Paulo SP, Brasil.

Correpondence:

João Carlos Papaterra Limongi;

Rua Bueno Brandão, 444 / Cob 1B;

04509-021 São Paulo SP, Brasil;

E-mail: limongi@uol.com.br

Conflict of interest:

There is no conflict of interest to

declare.

Received 01 June 2016

Accepted 08 June 2016
Neurodegeneration with Brain Iron Accumulation comprises a group of rare, genetic neurological disorders characterized by abnormal accumulation of iron in the basal ganglia, substantia nigra, thalamus and cerebellum. The globus pallidus is the most affected structure. The exact relationship between iron accumulation and the symptoms of NBIA is not fully understood. NBIA is a progressive condition and there is no known cure at this time ${ }^{1}$.

Until recent years, NBIA was known as Hallervorden-Spatz disease. This eponym has been abandoned after the discovery of the involvement of these two neuropathologists with unethical procedures during the Nazi regime ${ }^{2,3}$.

All forms of NBIA were included under the Hallervorden-Spatz eponym until 2001, when the first NBIA gene was discovered. That particular gene was found to cause the most common form of NBIA, the Pantothenate Kinase-Associated Neurodegeneration (PKAN)4.

Over the years, more genes mutations and new phenotypes have since been recognized. In 2006, a mutation in the PLA2G6 gene was discovered and related to the PLA2G6-Associated Neurodegeneration (PLAN) and included the already known Infantile neuroaxonal dystrophy. In 2011, the gene C19orf12 was identified as being responsible for Mitochondrial-membrane Protein-Associated Neurodegeneration (MPAN). More recently, another disorder was put under the NBIA spectrum, the Beta-propeller Protein-Associated Neurodegeneration (BPAN). These four subtypes of NBIA are considered the most frequent and are identifiable by their varying symptoms and associated gene changes ${ }^{5}$.

Six other rarer disorders have also been identified and included in the NBIA spectrum. These conditions are neuroferritinopathy, aceruloplasminemia, Kufor-Rakeb disease, Fatty Acid Hydroxylase-associated Neurodegeneration (FAHN), Woodhouse-Sakati Syndrome and COASY Protein-Associated Neurodegeneration (CoPan) $)^{5}$.

When a genetic origin cannot be identified with certainty, a patient may be diagnosed as having idiopathic NBIA. This may be specially challenging if a person is the first and only affected individual in the family. Even if there is a likelihood that a recessive mode of inheritance is involved, the diagnosis will depend on brain imaging and specific genetic studies.

The course of the disease is highly variable. Onset ranges from infancy to adulthood. Progression can be rapid or slow with long periods of stability. Symptoms may vary greatly probably due to different genetic mechanisms present in the various forms of NBIA spectrum but the factors influencing disease severity and the rate of progression are still unknown.

The hallmark clinical manifestations of NBIA are progressive dystonia and dysarthria, spasticity, parkinsonism and cognitive decline. Retinal degeneration and optic atrophy are often present. Besides iron accumulation, neuropathologic findings include axonal spheroids in the central nervous system and, in some types, in peripheral nerves.

The most common type of NBIA is Pantothenate Kinase-Associated Neurodegeneration (PKAN). The phenotypic continuum includes classic PKAN (early onset and rapid progression) and atypical PKAN (later onset and slower progression). Children with PKAN typically manifest gait abnormalities around age three years and later develop progressive dystonia, dysarthria, rigidity, spasticity, hyperreflexia, and Babinski sign. Individuals with later-onset PKAN are likely to present with speech difficulty; psychiatric symptoms are more frequent in the later onset form. In brain MRI, the eye-of-the-tiger sign, $\mathrm{T}_{2}$-weighted hypointense signal in the 
globus pallidus with a central region of hyperintensity, is virtually pathognomonic for PKAN.

In other forms of the spectrum, establishing a diagnosis of a specific NBIA disorder may be more challenging. Because brain MRI findings in NBIA evolve over time, it may be difficult to determine the diagnosis and establish the specific NBIA disorder, even years after the onset of symptoms. Once abnormal brain iron accumulation is identified, the various types of NBIA can then be considered. In both PLAN and Kufor-Rakeb syndrome, iron accumulation only develops in a portion of cases or may present later in the disease course ${ }^{6}$.

Ideally the diagnosis of an NBIA disorder is confirmed with molecular genetic testing, either with single gene testing by NBIA type or an NBIA multi-gene panel may be used. Molecular genetic testing of PANK2 identifies biallelic mutations in the majority of individuals with the eye-of-the-tiger sign on $\mathrm{MRI}^{7}$.

On the other hand, some of the rarest forms of NBIA should be distinguishable based on findings unique to each specific subtype: for instance, a slowly progressive course with cognitive decline and more prominent spasticity are indicative of MPAN; developmental delay in childhood with adult-onset dystonia-parkinsonism and dementia are specific to BPAN; autosomal dominant neuroferritinopathy may be identified by pedigree analysis and by the unique feature of cystic degeneration in globus pallidus and putamen; aceruloplasminemia is suggested based on the findings of diabetes mellitus and iron accumulation in the liver.

In view of the complexities of the various clinical syndromes and patent overlapping between then, to reach a specific diagnosis is particularly challenging. In the article by Salomão et al. ${ }^{8}$, in this issue, the authors provide a comprehensive review of the NBIA spectrum along with detailed clinical and radiological features that help to distinguish among the various types. Genetic tests, when available, should aid in the diagnosis. The present article is a useful guide for a proper diagnostic approach for NBIA cases, from clinical features and brain imaging findings to the genetic etiology.

\section{References}

1. Hogarth P. Neurodegeneration with brain iron accumulation: diagnosis and management. J Mov Disord. 2015;8(1):1-13. doi:10.14802/jmd.14034

2. Hayflick SJ, Westaway SK, Levinson B, Zhou B, Johnson MA, Ching $\mathrm{KH}$ et al. Genetic, clinical, and radiographic delineation of Hallervorden-Spatz syndrome. N Engl J Med. 2003;348(1):33-40. doi:10.1056/NEJMoa020817

3. Hallervorden J, Spatz H. Eigenartige erkrankung im extrapyramidalen system mit besonderer beteiligung des globus pallidus und der substantia nigra: Ein beitrag zu den beziehungen zwischen diesen beiden zentren. Z Gesamte Neurol Psychiatr. 1922;79(1):254-302. doi:10.1007/BF02878455

4. Kurian MA, McNeill A, Lin JP, Maher ER. Childhood disorders of neurodegeneration with brain iron accumulation (NBIA). Dev Med Child Neurol. 2011;53(5):394-404. doi:10.1111/j.1469-8749.2011.03955.x
5. Schneider SA, Dusek P, Hardy J, Westenberger A, Jankovic J, Bhatia KP. Genetics and pathophysiology of neurodegeneration with brain iron accumulation (NBIA). Curr Neuropharmacol. 2013;11(1):59-79. doi:10.2174/157015913804999469

6. Tonekaboni SH, Mollamohammadi M. Neurodegeneration with brain iron accumulation: an overview. Iran J Child Neurol. 2014;8(4):1-8.

7. Amaral L, Gaddikeri S, Chapman PR, Roy R, Gaddikeri RS, Marussi Vh et al. Neurodegeneration with brain iron accumulation: clinicoradiological approach to diagnosis. J Neuroimaging. 2015;25(4):539-51. doi:10.1111/jon.12195

8. Salomão RPA, Pedroso JL, Drumond MT et al. A diagnostic approach for neurodegeneration with brain iron accumulation: clinical features, genetics and brain imaging. Arq Neuropsiquiatr 2016;74(7):587-96. doi:10.1590/0004-282X20160080 